\title{
Actuales Políticas Públicas para la Desinstitucionalización y Rehabilitación de Personas Psicóticas en Chile. Experiencia de la Comunidad Terapéutica de Peñalolén
}

\section{Current public policies for the uninstitutionalization and rehabilitation of psychotics in Chile. The experience of the therapeutic community in Peñalolen}

\author{
Felipe Diaz', Javiera Erazo ${ }^{2}$, Carla Sandova ${ }^{3}$
}

\begin{abstract}
Resumen
En el presente trabajo se revisan críticamente las políticas públicas del Estado chileno en los últimos años en relación a la salud mental, específicamente aquellas referidas a la desinstitucionalización y rehabilitación de personas psicóticas.

Se realiza una revisión del actual Plan Nacional de Salud Mental y Psiquiatría, haciendo énfasis en aquellos lineamientos orientados a una concepción biopsicosocial de las enfermedades mentales a través de tres ejes de discusión: familia, comunidad y red.

Por último, se revisa una experiencia de tratamiento institucional de las psicosis que incorpora los aportes del psicoanálisis para pensar la psicosis y su tratamiento posible y en donde estos dos ejes se toman como puntos de trabajo: subjetividad y política.

Palabras Clave: Políticas Públicas en Salud Mental, Procesos de Desinstitucionalización, Comunidad Terapéutica de Peñalolén.
\end{abstract}

\section{Abstract \\ In this paper we critically review the policies issued by the Chilean goverment in recent years in relation to Mental Health, specifically}

1 Lic. En Psicología. Comunidad Terapéutica Diurna de Peñalolén. elipediazarancibia@gmail.com

2 Lic. En Psicología. Comunidad Terapéutica Diurna de Peñalolén.javierazo@yahoo.com

3 Lic. En Psicología, Comunidad Terapéutica Diurna de Peñalolén. Sandoval.carla@ gmail.com 
those concerning to the uninstitutionalization process of Psychiatric patients and the rehabilitation of psychotic persons.

Initially, we will review the current National Plan for Mental Health and Psychiatry, emphasizing those guidelines that are oriented towards a biopsychosocial conception of mental illness, represented in what we have defined as three major points of discussion: family, community and network.

Finally, we will report an experience of institutional treatment of psychosis that incorporates the contributions of psychoanalysis to conceptualize psychosis and its possible treatment, where subjectivity and politics are taken into account as major focal points.

Key Word: Public Policies of Mental Health, Uninstitutionalization process, Comunidad Terapéutica Diurna de Peñalolén. 


\section{Introducción}

\section{Evaluación del estado actual de las políticas en Salud Mental en Chile desde una perspectiva político-institucional}

A ocho años de la puesta en marcha del Plan Nacional de Salud Mental y Psiquiatría impulsado por el Ministerio de Salud, su implementación comienza a ser objeto de una serie de evaluaciones críticas proveniente de diversos actores públicos.

El cambio de modelo implicó no sólo una modificación de la gestión en salud por parte del Estado en respuesta a las nuevas necesidades de la población, sino también la instauración de una serie de ideas y conceptos fundados en el resguardo de los derechos humanos de los pacientes psiquiátricos que no han sido fáciles de asimilar por la institucionalidad psiquiátrica más tradicional en Chile. Pero no sólo estas voces son críticas al proceso; el mismo Ministerio en conjunto con los sectores que se encuentran a favor de la implementación del nuevo modelo, están concientes de que, en efecto, este Plan no se ha desarrollado exento de dificultades. Las opiniones apuntan a que éstas se deben principalmente a la falta de preparación de los funcionarios, pocas instancias para que los pacientes encuentren un hogar en la sociedad y, en general, al escaso financiamiento estatal (La Nación, 27 de julio del 2008).

Por otra parte, el departamento de Salud Mental (SM) de la Subsecretaría de Salud Pública del Ministerio de Salud, en conjunto con la Organización Mundial de la Salud (OMS), el año 2006 publicó un informe de evaluación del sistema de SM en Chile, a partir de la información obtenida por la aplicación del Instrumento de Evaluación de los Sistemas de Salud Mental de la OMS (WHO-AIMS). Dicho informe da cuenta del estado actual del sistema de Salud Nacional, lo que permite considerar en términos técnicos de qué manera el Plan ha dado cuenta de sus objetivos iniciales.

Si bien el objetivo principal de promover la desinstitucionalización de los pacientes crónicos mediante la implementación de instancias ambulatorias de tratamiento ha tenido importantes avances, existen muchos 
aspectos en el que el Plan no ha podido cumplir con sus expectativas (Informe WHO-AIMS, 2006).

Efectivamente, según este informe, el presupuesto total en salud pública ha aumentado a $2,14 \%$ con respecto del 1,2\% existente hasta el año 1999. Este aumento de recursos se ha visto acompañado por la disminución del gasto asignado a los hospitales psiquiátricos, los cuales actualmente reciben un 33\% del total del presupuesto, a diferencia del 57\% asignado el año 1999. Estos recursos han sido reconducidos a instancias de atención ambulatoria y de trabajo comunitario desde un enfoque biopsicosocial. El informe muestra que este aumento está lejos del 5\% necesario presupuestado por el Plan Nacional para funcionar al año 2010. Esta situación implica una gran dificultad para el desarrollo de sus objetivos, siendo un tope para la capacitación y aumento de los recursos humanos necesarios en el desarrollo de mayores instancias para la descentralización y el tratamiento ambulatorio, para la promoción de la integración de organizaciones y actividades comunales, y en el refuerzo de los recursos operacionales, especialmente los fármacos.

Junto al problema económico, la evaluación da cuenta de un vacío interno a la formulación del propio Plan con respecto a la legislación y a los derechos humanos. En esta materia sólo se cuenta con el Reglamento para la Internación de las Personas con Enfermedades Mentales y sobre los Establecimientos que la Proporcionan, puesto en marcha el 2001. Según indica el informe, "Los avances alcanzados por el Plan Nacional de Salud Mental, en la provisión de servicios y en el desarrollo de una red ambulatoria y comunitaria, pierden efectividad al dejar a las personas con enfermedades y discapacidades mentales desprovistas de protección de sus derechos esenciales, en un medio social estigmatizador y discriminador" (Informe WHO-AIMS, 2006, p. 57), siendo Chile uno de los pocos países en el mundo que no cuenta con una legislación específica en salud mental.

El siguiente trabajo buscará discutir críticamente las consideraciones epistemológicas con respecto a la locura que se encuentran contenidas en el Plan Nacional, desde una perspectiva política, institucional y clínica, con el fin de evaluar si los supuestos lógicos con los que el Plan entiende el proceso de desinstitucionalización implican una superación de la con- 
cepción objetivante de la locura que históricamente ha constituido el saber psiquiátrico.

Esta perspectiva crítica se concretizará en la presentación de una experiencia de trabajo clínico institucional con la psicosis cuya propuesta de abordaje terapéutico permite entrar en diálogo con los principios y objetivos propuestos por el servicio de salud. La experiencia de la Comunidad Terapéutica Diurna de Peñalolén, permitirá abrir una discusión, tanto a nivel epistemológico como a nivel de las actuales condiciones materiales que delimitan un posible tratamiento ambulatorio que en su hacer sostiene un diálogo con las institucionalidades de salud pública.

\section{Antecedentes Contextuales}

\section{Políticas Públicas e Institucionalidad en Chile en Salud Mental (SM). Iniciativas previas al Plan Nacional de Salud Mental y Psiquiatría del año 2000}

Revisaremos de manera sucinta algunos hitos que marcan la preocupación por institucionalizar las problemáticas de Salud Mental en Chile. Cabe destacar que previo a la década del 60 existen muy pocos hechos que den cuenta de un interés por parte del estado por institucionalizar y regular los cuidados requeridos por personas con trastornos psiquiátricos severos, así como por establecer programas de Salud Mental para la población general.

En 1966 se formula el primer Programa Nacional de Salud Mental. Sin embargo, éste corresponde más a una declaración de intenciones que a una realidad objetiva. Luego de este intento, a partir de 1968, un equipo liderado por el Dr. Juan Marconi establece varios programas de atención organizados bajo los lineamientos de la psiquiatría comunitaria, que funcionan con la participación de diversos profesionales de SM y actores comunitarios que buscan reemplazar el modelo asilar. Posteriormente a 1973 estos proyectos son terminados abruptamente y no es hasta el reestablecimiento de la democracia que se vuelve a poner en la agenda del Ministerio de Salud los problemas en SM y las críticas a los modelos centrados en el 
hospital psiquiátrico. Entre las primeras acciones está la instauración de un Plan Nacional de Salud Mental y Psiquiatría, el cual es antecedente directo del Plan actual.

El año 1998, en el Decreto Supremo N ${ }^{\circ}$ 570, llamado Reglamento para la Internación de las Personas con Enfermedades Mentales y sobre los Establecimientos que la Proporcionan, se legaliza la necesidad de garantizar los derechos de las personas hospitalizadas sin su consentimiento y se regulariza los protocolos de atención y las medidas de protección que se deberán cumplir en el lugar de internación, impidiendo abusos y violaciones a los derechos de los pacientes por parte del equipo que los atiende. Este mismo decreto instaura la creación de la Comisión Nacional de Protección de las Personas Afectadas de Enfermedad Mental. Si bien el Decreto se estableció en el año 1998, éste no entró en vigencia hasta el de 14 de Enero del 2001, al año siguiente del establecimiento del Plan actual.

\section{Antecedentes Internacionales}

El actual Plan se organiza de acuerdo a las directrices establecidas por la Organización Mundial de la Salud (OMS). Estos lineamientos están establecidos en la Declaración de Caracas, la cual Chile suscribió en 1990. Ahí se señala la necesidad de los países Latinoamericanos de establecer políticas, planes y programas explícitos para lograr responder a las necesidades de atención en Salud Mental de su población. Los valores promovidos por la Declaración fueron la permanencia del enfermo en su comunidad, la promoción de servicios de atención psiquiátrica ligada a la Atención Primaria en Salud (APS) y el respeto por los Derechos Humanos (Minoletti, A., 2005).

\section{Plan Nacional de Salud Mental y Psiquiatría (2000)}

Bajo la necesidad de cumplir los compromisos asumidos con la Declaración de Caracas, este Plan busca rescatar la preocupación por la SM y el modelo comunitario característico de los años 60 , pese a que sus propuestas y modelos de intervención no dan cuenta del trabajo realizado en esos años. 
El Plan ha establecido ciertos principios rectores que dan cuenta de sus valores fundamentales. En primer lugar, la concepción de la salud, y en especial de la SM, no es dependiente sólo de factores biológicos sino también psicológicos y sociales. Por esto las condiciones en que viven y se desenvuelven los sujetos son de vital importancia para la mantención del bienestar y para minimizar la discapacidad y marginación social de aquellos afectados por un trastorno psiquiátrico. La identidad y sentido de pertenencia son considerados factores protectores de la salud; por lo tanto, las atenciones deben centrarse en los espacios que habitan los sujetos. En segundo lugar, la atención deberá ser siempre de la mejor calidad humana y técnica. Por otra parte, se propone prestar atención de acuerdo a un enfoque comunitario, en una red establecida de SM y psiquiatría, que trabaje de manera coordinada con la red de APS. Además, busca integrar y asegurar intervenciones en promoción, prevención, tratamiento y rehabilitación. Por último, intenta renovar y prestar atenciones de acuerdo a datos actualizados con respecto al costo-efectividad de las intervenciones.

Si bien el Plan representa las directrices de intervención y se respalda y organiza de acuerdo a varios documentos oficiales del Ministerio de Salud, no tiene ninguna representación en la legislación actual. Tiene carácter de documento técnico, por tanto sus lineamientos y continuidad no están garantizados (Minoletti, A. y Zaccaría, A., 2005).

Como habíamos anticipado, el Plan organiza sus estrategias de acuerdo a objetivos establecidos por la OMS en tres líneas prioritarias:

\section{-Abordaje Comunitario.}

Se entiende por enfoque comunitario un modelo que privilegia las prestaciones ambulatorias de atención, en dispositivos ubicados en los territorios que habitan los usuarios, que se encuentran organizados en red para proveer una atención coordinada y adecuada no sólo a las necesidades de cada usuario, sino también a las características psicosociales de una determinada población. Este modelo privilegia la reintegración social y el fortalecimiento de los lazos del usuario con su comunidad.

Se busca también su enriquecimiento en función de actividades que impliquen a los dispositivos locales rescatando la experiencia de quienes 
trabajan en las comunidades. Por otra parte, el abordaje comunitario tiene como foco principal involucrar la participación del paciente, sus familiares y actores comunitarios significativos. Se espera incentivar y dar aporte financiero a las agrupaciones de usuarios y familiares para reforzar sus capacidades de autoayuda, facilitarles el control y colaboración con respecto a la calidad de atención, la protección de los derechos de los pacientes (Ministerio de Salud, 2000, Cap. 5, p. 2)

\section{-Atención Psiquiátrica en relación a Atención Primaria en Salud}

Este cambio busca aumentar la cantidad de prestaciones y la accesibilidad a las atenciones en SM. Para esto sería necesario aumentar la capacidad resolutiva en este nivel. Esto se ha logrado aumentando el número de horas de diversos profesionales, como psicólogos, psiquiatras, médicos con capacitación en SM e implementando consultorías en APS (supervisión por parte de psiquiatras del nivel secundario y terciario de casos de SM atendidos en APS).

Para implementar estos desafíos se han reconvertido algunos recursos del hospital psiquiátrico hacia la atención primaria, lo que ha sido acompañado de una disminución de las camas y atenciones psiquiátricas que se realizaban en el hospital.

La atención se debe organizar en red de manera coordinada con los dispositivos especializados, de manera de priorizar la promoción, la prevención y la atención temprana. "Los distintos componentes de la red deben articularse coherentemente de modo de asegurar la diversidad e intensidad de las atenciones requeridas por el paciente y su familia, la continuidad de los cuidados, la existencia de distintos niveles de resolución, y el acceso a medidas de promoción y protección de la salud" (Ministerio de Salud, 2000, Cap. 5, p. 1).

\section{-Respeto a los Derechos Humanos}

Además de la protección a los pacientes establecida en el Decreto No 570, surgen en el año 2005 las Garantías Explicitas en Salud (GES), las cuales implican acceso, oportunidad, protección financiera y calidad de atención; todos estos entendidos como derechos fundamentales. En una primera etapa fueron priorizadas 3 patologías en SM: Esquizofrenia Primer 
Episodio, Depresión en personas de 15 años o más, y Consumo perjudicial o dependencia a alcohol en menores de 20 años.

Por otra parte, con la noción de ciudadanía, se pone énfasis en los derechos a la inclusión social y a la no marginación; buscando reposicionar en los tratamientos la lucha por los derechos cívicos y de participación de los usuarios. 'La enfermedad mental implica, entre muchas otras cosas, la pérdida del lugar que la persona afectada ocupaba en la sociedad. Por lo tanto, la recuperación de la capacidad de ejercer los derechos de ciudadanía constituye uno de los puntos esenciales para evitar la estigmatización de estas personas y atenuar el riesgo de exclusión socio comunitaria." (Ministerio de Salud, 2005. p. 26).

Luego de este recorrido, nos centraremos en aquellas orientaciones técnicas referidas a la atención y tratamiento de los trastornos psiquiátricos severos y esquizofrenia, poniendo atención en las intervenciones psicosociales, especialmente en la noción de rehabilitación.

\section{Estrategias y Orientaciones Técnicas para la atención de Esquizofrenia y Trastornos Psiquiátricos Severos}

Las nociones de trastorno psiquiátrico severo y esquizofrenia presentes tanto en la Norma Técnica como en el Protocolo GES siguen la línea de las clasificaciones aceptadas mundialmente (CIE-10 y DSM-IV) y además resalta el efecto que tienen en el ámbito social donde surgen. "La esquizofrenia es una condición crónica que frecuentemente tiene efectos devastadores en muchos aspectos de la vida del paciente y conlleva un alto riesgo de suicidio y el menoscabo de la vida de la persona. Las consecuencias de la enfermedad no son sólo individuales sino que afectan en forma dramática al entorno familiar y social inmediato y generan altos costos económicos para el país" (Ministerio de Salud, 2005, p.5.)

El enfoque de intervención propuesto para estos usuarios implicaría una complementación del tratamiento biológico con intervenciones de tipo psicosocial, pues este modelo habría demostrado sistemáticamente el mayor nivel de efectividad. Además, los programas de mayor costoefectividad estarían basados en estrategias comunitarias, entendidas como 
“... el modo de trabajar las intervenciones en salud mental, siendo el área comunitaria el contexto general donde se desarrollan las intervenciones. La premisa básica es impedir la desvinculación social de la persona. De esta forma el sujeto mantiene un rol social valorado, se evita el deterioro de sus habilidades y se minimiza la discapacidad" (Ministerio de Salud, 2005, p.33). Por otra parte, se consideran las acciones psicosociales “...realizadas por el equipo de salud mental tendientes a facilitar la integración del individuo afectado por la enfermedad a su entorno natural." (Ministerio de Salud, 2005, p.33) Estas acciones incluyen: psicoeducación, psicoterapia, intervenciones familiares, manejo ambiental y rehabilitación.

El concepto de Rehabilitación incluye la intervención en actividades de la vida cotidiana, entrenamiento en habilidades sociales, rehabilitación vocacional y utilización del tiempo libre. La posibilidad de pensar la rehabilitación como una intervención adecuada para las personas que sufren de enfermedad mental está relacionada con el desarrollo del concepto de discapacidad de causa psíquica, pues éste, de acuerdo a las nuevas normas, “...ha permitido dejar de lado el prejuicio existente respecto de la persona con esquizofrenia como alienada y sin contacto alguno con sus afectos y la sociedad que la rodea y destacar que la esquizofrenia es una condición que incapacita de un modo variable algunos aspectos de la vida de la persona y que, por lo tanto, es posible establecer estrategias para superar esas limitaciones" (Ministerio de Salud, 2005, p.24).

Los esfuerzos de desinstitucionalización, mediante la instauración de políticas de inclusión y promoción de la rápida reinserción a la comunidad y a la familia en los primeros años de enfermedad, son efecto de estas propuestas de intervención.

Paralelamente al trabajo de definición de los aspectos técnicos presentes tanto en el Plan como en las Orientaciones Técnicas, que congregan intervenciones tanto clínicas como de rehabilitación, el Ministerio de Salud ha avanzado en la definición de los costos que implican estas prestaciones específicas. La definición oficial, operativa a partir del año 2002 y regulada por la Ley 18.469, está dada por las prestaciones FONASA en la modalidad de atención institucional en Salud Mental y Psiquiatría y se denominan: "Programas de Rehabilitación Tipo 1 y Tipo 2". Éstos contemplan 
una serie de intervenciones psicosociales y médicas con el usuario y su familia. Ambos programas consisten en una provisión regular y continua de un conjunto de actividades tales como acompañamientos terapéuticos, evaluación de discapacidad, diseño de un plan terapéutico, sesiones individuales con psicólogo y terapeuta ocupacional, intervenciones grupales, asistencia a la familia, apoyo a la reinserción social y laboral, entre otros. Ambas prestaciones son otorgadas al paciente por un equipo de trabajo integrado preferentemente por psiquiatra, psicólogo, terapeuta ocupacional, enfermera, asistente social, técnico y/o monitor, pertenecientes al equipo ambulatorio de SM y psiquiatría.

Actualmente la valoración FONASA para ambos Programas, corresponde a $\$ 2.590$ y $\$ 6.070$ diarios, respectivamente, por paciente. De esta manera se definen las horas de destinación de los equipos y los costos asociados para su implementación por parte de las instituciones públicas y privadas que se vinculan al Estado y que toman en tratamiento ambulatorio a personas psicóticas. Según Saldivia (2004), existen bajo esta modalidad de financiamiento, en la región metropolitana, un total de 17 Programas de este tipo, siendo la Comunidad Terapéutica Diurna de Peñalolén la más antigua, y actualmente una entre cuatro llevadas a cabo en el área oriente de Santiago.

\section{Análisis: \\ Desinstitucionalización: Complementación Biopsicosocial del Modelo Médico}

Dentro de los propósitos del Plan, se expresa con claridad el fundamento biopsicosocial del modelo que se encuentra a su base en los siguientes postulados:

La salud no sólo depende de factores inherentes a las personas, sino que también de las condiciones en que ellos viven.

Participación activa del paciente y sus familiares.

Reinserción social de personas con enfermedades mentales, evitando la estigmatización y marginación y creando redes de apoyo. 
De manera general, se establece que el proceso de desinstitucionalización constituye un esfuerzo importante por mejorar la calidad de vida de los pacientes psiquiátricos crónicos, mediante la restitución de sus derechos ciudadanos para su completo desarrollo humano en el exterior del hospital. Es un esfuerzo, entonces, por asegurar la inclusión de estos pacientes a través de un proceso de rehabilitación psicosocial. Dicho proceso implica una superación de la lógica médica tradicional representada por la figura de la internación en el hospital psiquiátrico, mediante una complementación del saber médico con los aportes desarrollados desde las ciencias sociales. Esto implica la instalación de una concepción biopsicosocial de la enfermedad mental, donde la familia y lo comunitario son elementos fundamentales tanto para el mantenimiento como para la recuperación de la salud.

De esta forma, el retorno a la comunidad y a la familia no sólo le restituye a los pacientes sus derechos ciudadanos sino que también da paso a la restitución de su salud.

Existe el supuesto implícito de que los pacientes, al estar viviendo en sus comunidades de origen y contando con el apoyo y acompańamiento de sus familias, necesariamente obtendrían un efecto terapéutico, una mejora en el nivel general de su salud, razón por la cual son espacios privilegiados en el tratamiento: "La prevención, detección y tratamiento precoz y efectivo de los trastornos mentales requiere de acciones de salud que involucren a las personas, familias y grupos organizados de la comunidad, así como de servicios de otros sectores. Dado que la identidad y sentido de pertenencia son factores protectores, debe privilegiarse la atención más cercana a los espacios naturales de las personas y en el caso de necesitar atención cerrada esta deberá ser en instancias lo más parecidas posible a un ambiente familiar” (Ministerio de Salud, 2000, Cap 5.)

Entonces, cabe preguntarse cómo actúan estos factores protectores, qué es lo que hay de propiamente terapéutico tanto en la familia como en la comunidad. Es pertinente intentar reconocer en esto cuáles son los elementos que agrega, qué contrapone o qué sustituye la concepción biopsicosocial a la lógica médica.

Dentro de las acciones que recorren el proceso de desinstitucionalización que el Plan exige, consideramos que dentro de esta propuesta, y sobre 
todo en relación a los pacientes con diagnósticos de patologías psiquiátricas severas, las nociones de familia, comunidad y red aparecen concretamente en relación a la importancia terapéutica de los aspectos sociales en el abordaje psiquiátrico, que se presentan como novedad respecto a su propia tradición institucional. Consideramos pertinente problematizar estos puntos.

Se podría pensar que el papel terapéutico de la familia, de la comunidad, de las redes sociales y de la propia red de salud, no tienen que ver necesariamente con cierta especificidad de cada una de ellas, sino más bien con permitir anular los efectos institucionalizadores del hospital, convirtiéndose en alternativas que vuelven innecesario el encierro.

$\mathrm{Al}$ menos en lo que respecta a la familia, esto es cuestionable. De ser así, cualquier instancia de acogida en el exterior de la comunidad tendría el mismo valor para las indicaciones del Plan. Pero es fácil reconocer que en la planificación de estos sistemas de acogida para la desinstitucionalización, la familia tiene un rol primordial. Dispositivos como los hogares o las residencias protegidas sólo tienen una prioridad menor, son considerados como alternativas sólo en segunda instancia; una vez que las familias no han sido capaces de tomar los pacientes a su cargo.

\section{Familia}

El Plan contiene una recurrentemente apelación a la participación activa de la familia en el proceso de recuperación de los pacientes. Es fundamental que la familia asuma su responsabilidad con aquellos miembros que necesiten de cuidados psiquiátricos, brindándoles apoyo y acogida, intentando comprender con ayuda de los profesionales de SM que los problemas presentados responden principalmente a su condición patológica. Implícitamente, la acogida familiar es una condición para la recuperación de la locura, aunque no queda claramente definido en qué sentido.

Si consideramos que para Michel Foucault (2005), la familia y la institución psiquiátrica clásicamente identificada con el hospital, mantienen una estrecha relación, la circulación de los pacientes de uno de estos lugares a otro no deja de ser problemática.

Esta relación estrecha se reconduce al vínculo entre la formación de la familia moderna y el saber médico. Según Foucault (2002a), la familia 
moderna se establece como un recorte en términos cuantitativos, y una modificación en términos cualitativos, de las grandes familias predominantes hasta mediados del siglo XVII. Este nuevo cuerpo familiar, esta familia-célula comienza a constituirse como núcleo restringido, delimitado por una relación estrecha entre los padres y los hijos. La familia, desde ahí en adelante, cumplirá la función de preparar y resguardar la inscripción de los sujetos al interior de los dispositivos disciplinarios como la escuela o el trabajo, instancias que producen y reproducen un determinado orden social. La familia es la estructura fundamental, "el punto de enganche absolutamente indispensable para el funcionamiento mismo de todos los sistemas disciplinarios... es la instancia de coacción que va a fijar de manera permanente a los individuos a los aparatos disciplinarios, que en cierto modo va a inyectarlos en ellos" (Foucault, M., 2005, p. 105). Situación por la que no es posible desconocer que toda apelación a la familia implica la consideración del funcionamiento de una modalidad de control social.

Pero la familia tiene una función "bisagra", en la medida que sostiene los sistemas disciplinarios, pero a la vez se constituye como objeto mismo de su funcionamiento toda vez que no es capaz de asegurar esta tarea: "cuando la familia se hace trizas, cuando deja de cumplir su función, no tarda en introducirse toda una serie de dispositivos disciplinarios cuyo papel consiste en mitigar sus flaquezas" (Foucault, M., 2005, p.108.). Es aquello que Foucault denomina "función psi", las tecnologías que se activan operando sobre la familia. La función psiquiátrica, psicológica, psicoterapéutica, psico-sociológica, criminológica, corresponden a la organización de un dispositivo que se precipita en un intento de normalización técnica frente a cierta anomalía, ante la cual la soberanía familiar no puede responder. Para esto, dichos dispositivos refieren siempre a la familia, intentan restituir su orden, recobrar su importante funcionamiento.

Así, psiquiatría y familia se refieren mutuamente. Se filtra en la familia un saber sobre la locura, siempre referido a la familia, que implica una tecnificación proveniente desde el saber psiquiátrico y las tecnologías que le sirven de apoyo. Un "saber hacer" con los locos, con su enfermedad, que le permite a la familia estar con él, acompañarlo, conducirlo, aceptarlo y contenerlo. 
Esta vinculación intrínseca da cuenta de la función de control que la familia tiene respecto del paciente. Se constituye como un espacio de contención para ellos, espacio rodeado y asistido por una serie de tecnologías referidas al saber psiquiátrico, que amortiguan dicha función.

\section{Comunidad}

A pesar de que en el Plan todo apunta a la reinserción y el retorno de los pacientes a sus comunidades de origen, parece no existir una delimitación clara de lo que implica el concepto de comunidad.

En efecto, este concepto aparece mayormente referido al lugar originario de los pacientes, el espacio exterior al hospital. En relación a esto, se considera también que es el lugar donde deberán apuntar las intervenciones necesarias para brindar una máxima cobertura de salud a la población. La comunidad será el lugar donde se instalarán los centros de salud necesarios para la atención médica, psiquiátrica y psicológica; será también objeto de potenciación, donde se propiciará la autogestión de espacios colectivos que permitan el resguardo de la salud, como también la completa reinserción de los pacientes crónicos institucionalizados.

En todas las acciones referidas a la comunidad, ésta siempre queda sin definir. No se da cuenta de sus especificidades, de su lugar social, de sus relaciones administrativas o de las instituciones que allí operan (Foladori, H. 2005). Solamente indica un territorio físicamente delimitado, sin referencia a los criterios que establecen dicha limitación. En última instancia, corresponde a un espacio territorial externamente definido, que engloba un conjunto preciso de sujetos, a quienes se les garantiza una serie de instancias que les permiten satisfacer sus necesidades de SM.

Maritza Montero (2004) entiende el concepto de comunidad más allá de toda delimitación externa. Se encuentra autodelimitado por la comunidad misma; como sujeto activo de lo que se lleva a cabo dentro de ella; como actor social constructor de su propia realidad.

En este sentido, llama la atención que no exista referencia alguna dentro del Plan a lo que los sujetos que la componen dicen de sí mismos en relación a esta denominación, a algún tipo de organización interna en función de sus propias necesidades, a espacios propiamente gestionados 
como búsqueda de satisfacción de sus demandas. Cualquier referencia a la autogestión es siempre externa, bajo la forma de "propiciarla", lo cual en definitiva, corresponde a una contradicción (Foladori, H., 2005).

Se desprende de los conceptos del Plan que no existe nada "común" a los habitantes de una comunidad, más que estar definidos por su coexistencia al interior de un recorte espacial.

Ante esta situación, parece problemático que se organicen en torno a ella una serie de intervenciones que tienen como objeto lo comunitario. El llamado "enfoque comunitario" no cuenta con más especificidad que ser una serie de prácticas que se desenvuelven al interior de un territorio. $\mathrm{Al}$ ser su objeto necesariamente difuso, también lo serán sus prácticas, sin referencia a una delimitación teórica clara.

Concretamente se puede observar que dentro de las intervenciones comunitarias caben todos tipos de acciones, provenientes de marcos conceptuales diversos y hasta contradictorios, que no cuentan necesariamente con una coherencia teórica que las sostenga, en una integración ecléctica que desconoce las implicancias epistemológicas y políticas de sus acciones.

Como apunta Foladori (2005) con respecto a la psicología comunitaria, la inespecificidad del enfoque comunitario, al poner el acento en lo común externamente definido, borra las particularidades al interior de cada comunidad, y también las diferencias entre distintas comunidades; particularidades que tienen relación con diferencias al interior de una estructura social, con un "ejercicio (apropiación) del poder" (p.132).

Nuevamente, la referencia a lo social al interior del Plan, más allá de las buenas intenciones, se topa con la instalación de un marco conceptual que invisibiliza una serie de dificultades que tienen efectos concretos en su aplicación.

\section{Red de Salud Mental y Psiquiatría}

El cambio hacia un modelo en el que el eje ya no es la estructura física del establecimiento hospitalario, implica la planificación, organización y coordinación de instancias descentradas del hospital, donde cada una se encuentra técnicamente capacitada para que, en coordinación con 
las demás, puedan cumplir con las diversas actividades de SM que el Plan propone. Todo esto, a partir de que la evidencia indica que como dice el Plan, "la estrategia basada en redes de atención que integren en forma coherente todos los dispositivos necesarios - desde el tratamiento hospitalario intensivo hasta dispositivos intracomunitarios de apoyo- optimizan la utilización de los recursos y mejoran significativamente las consecuencias del trastorno y la calidad de vida de las personas" (Ministerio de Salud, 2000. Cap. 5). De esta forma, enfatiza el impacto en la dimensión social de los tratamientos, situación que anteriormente era minimizada en relación a lo propiamente médico.

En este sentido, en lo que el Plan define como patologías psiquiátricas severas el tratamiento pensado para estas afecciones se modifica fundamentalmente a partir del concepto de red.

Los pacientes que caen bajo esta denominación, son precisamente aquellos que durante mucho tiempo fueron sostenidos por el hospital psiquiátrico. El concepto de prestaciones implica un quiebre en la necesidad de internación en estos pacientes; instala la posibilidad de entregar solamente las atenciones necesarias para contrarrestar los efectos de la patologías en aspectos puntuales de sus vidas. Esto desdibuja la exigencia de un espacio que aborde la totalidad de la vida de estos pacientes. Así, las prestaciones logran fragmentar los tratamientos necesarios que garanticen los cuidados a los enfermos, de tal manera que deja fuera de la preocupación psiquiátrica los aspectos de la vida cotidiana que no se ven afectados, y que anteriormente eran necesariamente administrados por el encierro.

Esta serie de prestaciones se encuentran distribuidos en el territorio, situados al interior de las comunidades, lo que implica que los pacientes se mantendrán lo más cercanamente posible de sus domicilios, sus vínculos significativos y su rutina diaria. Junto a esto, para dar respuestas a las necesidades de reinserción social, a los cuidados y garantías que demanda este proceso, se ha operacionalizado el concepto de "discapacidad de causa psíquica”, que establece un paralelismo con la discapacidad física, condición que sólo necesita de asistencia y no de encierro.

Pero más allá de estas modificaciones, cabe preguntarse si las prestaciones mismas ofrecen alguna novedad en su contenido, en la lógica que 
las recorren, en relación a las acciones clínicas que tienen lugar dentro del hospital psiquiátrico; es decir, más allá de la fragmentación y de la ubicación al exterior del espacio hospitalario, ¿̇las acciones clínicas de la red y la noción de discapacidad de causa psíquica, presentan alguna diferencia sustancial en el tratamiento de los pacientes psicóticos?

La apertura hacia la comunidad pone a la rehabilitación psicosocial como uno de los objetivos prioritarios a lograr en este tipo de pacientes. Este énfasis sitúa a este proceso y a las prestaciones necesarias para su consecución como una novedad del Plan respecto a las atenciones anteriores.

Según el Plan, los objetivos de estas intervenciones son la mantención de las habilidades sociales, académicas y profesionales; la redefinición de un proyecto de vida auto valorado y valorado por la comunidad; y el desarrollo de un rol o identidad positiva. De esta forma, la rehabilitación psicosocial es entendida no sólo como un conjunto de técnicas sino, como apunta el Plan, "una filosofía asistencial que ofrece una visión de la enfermedad mental en la cual la dimensión socio ambiental tiene tanta importancia como la visión biológica y donde la evaluación de la discapacidad y la minusvalía es tan importante como el tratamiento de los síntomas" (Ministerio de Salud, 2000), donde el manejo óptimo de la persona con discapacidad de causa psíquica requiere la integración del tratamiento médico con intervenciones psicosociales.

A partir de esto se presentan dos problemas. En primer lugar, de los objetivos de este proceso se desprende la necesidad de un determinado ajuste social en este tipo de pacientes, el cual se hace por medio de un trabajo sobre sus rutinas diarias, sus interacciones y en el espacio interior de sus expectativas de vida. Este ajuste se traduce nuevamente, al igual que dentro del hospital, en un control moral de los aspectos de su vida cotidiana que aparezcan siendo disruptivos en relación a ciertas expectativas sociales.

En segundo lugar, todo tipo de atención centrada en la dimensión socio ambiental debe estar siempre referida a la atención médica para poder sostenerse. Junto a esto, la noción de "discapacidad psíquica" sigue presentando a las personas psicóticas como aquellas a quienes les falta una determinada capacidad psíquica para operar con normalidad. Demarcación de una carencia, realiza tanto por el modelo médico como por el 
psicosocial, que sigue situando a estos pacientes dentro de la noción de enfermedad.

Así se observa que la noción de locura que sigue recorriendo las prestaciones de la red es la identificación con la enfermedad que desde Kraepelin en adelante, ha instalado el saber psiquiátrico.

Si bien la noción de red implica una importante modificación en la gestión estatal del problema de la Salud Mental, en términos epistemológicos la noción de enfermedad, propia del modelo médico, sigue estando inscrita en las prestaciones de la red. Pese a la inclusión del modelo psicosocial, éste aparece en referencia a las mismas nociones presentes al interior del saber psiquiátrico, lo que claramente no implica un cambio en lo que históricamente se ha entendido por locura en este contexto.

\section{Una experiencia de trabajo en rehabilitación: la Comunidad terapéutica de Peñalolén (CTP)}

La institución, de la cual describiremos algunos aspectos de su trabajo en tratamiento y rehabilitación de personas con diagnóstico de psicosis, se constituye en 1975, durante la dictadura militar en Chile, a partir de un grupo de profesionales y voluntarios que con apoyo de la Vicaría de la Solidaridad dan lugar a un policlínico Parroquial al alero de la Parroquia San Roque. La actividad está fuertemente vinculada al trabajo comunitario en salud y a la labor del voluntariado parroquial. Posteriormente, en los años 1989-90, con el aporte técnico y financiero de la Cooperación Italiana en Chile, el proyecto redefinió sus objetivos específicos hacia un "Programa de Atención Primaria de Salud con énfasis en Salud Mental”. El trabajo mantendrá el acercamiento activo de los profesionales con la población, por ejemplo, a través del vínculo con las juntas de vecinos, con quienes se realizan talleres y grupos de salud, capacitando a sus integrantes en el conocimiento de problemas psiquiátricos diversos que pudieran aquejar a personas de la comunidad. Por otra parte, se realizaban visitas "casa a casa" con el objeto de detectar personas con problemas de salud mental, específicamente psicóticas, que vivían en precarias condiciones (Hunneus, T., 2005). 
A partir de este trabajo, de acercamiento y cuidados cercarnos con personas psicóticas, se hizo necesario para el equipo formalizar estas acciones en un Programa de Rehabilitación Diurno Ambulatorio, tomando las experiencias de des-institucionalización de reforma en Italia e Inglaterra. Estas experiencias sirvieron de referente para el análisis crítico de las políticas de Salud Mental vigentes hasta los años '90 en Chile, basadas en el énfasis asilar y el tratamiento médico tradicional.

En 1991 el programa de rehabilitación psicosocial Comunidad Terapéutica de Peñalolén, asentado en la Parroquia San Roque, formaliza un convenio con el Estado. Este año la Cooperación italiana en Chile finaliza el apoyo económico, sostenido durante dos años, a la institución, en la medida que no sólo se consolida el retorno a la democracia sino también se comienza a gestar un discurso claro del lado del estado relativo a las políticas en salud mental acorde a los lineamientos internacionales. La disposición legal con que cuenta el ministerio para financiar este proyecto será el DFL 36 de 1990. Este es un convenio histórico que vincula tres instituciones volcadas a dar continuidad a un trabajo local: la vicaría, el municipio y el Ministerio de Salud. Hasta esa fecha no se contaba con nominación formal para este tipo de programas, es decir ni referencias técnicas ni financieras para el tratamiento ambulatorio para psicóticos, en su mayoría sin tratamiento y en condiciones sociales precarias.

De esta manera la institución se convierte en una de las pioneras en su aplicación nacional, no sólo en el establecimiento de un diálogo directo con el estado en tanto institución privada, sino también en la ejecución de un programa comunitario no médico de acompañamiento diario de psicóticos, que ha permitido prácticas concretas de desinstitucionalización psiquiátrica.

Cabe destacar que hasta el año 2001, el programa que sostiene, acompańa y da seguimiento a, en promedio, 30 usuarios, es co-financiada por el Ministerio de Salud y el Municipio local, en un 66\% y 33\% respectivamente. A partir del año siguiente hasta la fecha, el aporte local intersectorial no sobrepasa el 7\% del programa. Es llamativo que esta reducción de la participación local sea contemporánea con la definición de las prestaciones de salud FONASA (Ministerio de Salud, 2001). 
La Comunidad Terapéutica de Peñalolén desarrolla sus actividades en dos pequeñas casas ubicadas en una zona pobre de la comuna de Peñalolén, cercana a las poblaciones surgidas de tomas de terrenos. Cuenta con dos salas de trabajo grupal, un pequeño patio, además de tres salas de atención individual, tres baños, cocina y una oficina para las reuniones de equipo.

El equipo actualmente está constituido por dos terapeutas ocupacionales ( $44 \mathrm{hrs}$ ), tres psicólogos ( $44 \mathrm{hrs})$, tres monitores (12 hrs), una secretaria jornada completa, además de una decena de voluntarios, entre estudiantes en práctica, pasantes y otros monitores. Este equipo realiza el "Programa Regular de Rehabilitación", actual nomenclatura que le confiere el Plan a este tipo de tratamiento, para un cupo de 30 beneficiarios, de los cuales 17 son beneficiarios de un programa Tipo 1, y 13 son beneficiarios de un programa Tipo 2 de rehabilitación psicosocial. Estos cupos de Rehabilitación corresponden al actual convenio entre la institución y el Servicio Metropolitano Oriente; sin embargo, los usuarios que mantienen una relación con la institución superan las 50 personas.

El modelo de tratamiento considera el vínculo a variados espacios grupales e individuales, dependiendo de cada caso. Los usuarios reciben la indicación de hacerse parte de la cotidianeidad en la Comunidad a través de actividades grupales e individuales diarias, que estructuran los días, el tiempo y el espacio. Las actividades colectivas se organizan en dos asambleas comunitarias semanales, en las que se distribuyen los cupos de los diferentes talleres terapéuticos, artísticos y sociales; se discuten las dificultades propias a la convivencia, la distribución de responsabilidades, el manejo de asuntos del tiempo libre y económico, e informaciones o problemáticas sociales que ingresan al colectivo. Este grupo de trabajo es facilitado por el equipo. El tratamiento “individual” se inscribe en esta dinámica grupal, constantemente facilitada por el equipo y por los propios usuarios, bajo el supuesto de que es el espacio habitado ahí el que reproduce las condiciones facilitadoras o inhibidoras de su desarrollo como sujetos, y que de este modo es el medio principal de abordaje a las problemáticas asumidas colectiva y cotidianamente.

Nos parece pertinente discutir en este trabajo la experiencia institucional de Comunidad Terapéutica Peñalolén y la manera que esta ha 
encontrado para pensar la locura, gestionar los espacios colectivos de tratamiento, con el objeto de ilustrar las tensiones políticas-institucionales que anteriormente hemos discutido a partir de las políticas públicas y a las condiciones materiales posibles para desenvolver tratamientos institucionales relativos a la psicosis en Chile, resituando a partir de los siguientes aportes psicoanalíticos la pregunta por la psicosis entendida más allá de una enfermedad o déficit subjetivo.

\section{Pensar la institución: referencias teóricas psicoanalíticas que atraviesan una aproximación clínica de la locura}

Dentro de las referencias psicoanalíticas para pensar el trabajo cotidiano con la psicosis en la Comunidad Terapéutica Peñalolén, se cuentan los conceptos de: transferencia, discontinuidad del sentimiento de existir y el tratamiento por el colectivo.

El trabajo llevado a cabo estos años ha implicado entender al sujeto psicótico como sujeto con deseo; “...deseo inconsciente en el sentido de Freud, rearticulado de manera extraordinaria por Lacan. Hay deseo incluso en las personas supuestas como las más retrasadas, incluso en aquellas que tienen un IQ de 30, los idiotas, los cretinos. Hay deseo y es eso lo que está en cuestión: podemos decir es una opción ética. Entonces, si hay deseo, existe una dimensión psicoanalítica en el sentido de Freud, por mínima que ésta sea (...) pero a fin de cuentas hay una dimensión de reflexión que debe considerar el deseo inconsciente" (Oury, 1998).

La dimensión del deseo nos permite pensar y poner en cuestión el concepto de transferencia en la psicosis. Entendida desde sus orígenes por Freud "como una suerte de movimiento, de desplazamiento, incluso de investidura en el cuerpo"... "entonces hay movimiento: cuando decimos transferencia decimos movimiento, decimos deseo inconsciente" (Oury, 1998). Por lo tanto la noción de transferencia requiere de un espacio. Perdemos de vista, dice De Certeau (2003), que transferir implica necesariamente un espacio donde sea posible el desplazamiento de un lugar a otro. Algo debe desplazarse, de y por una realidad a otra. 
En este sentido, podemos pensar que la institución se dispone como un lugar que tiende a la creación de espacios e instancias investibles y diferenciables.

Siguiendo a Pereda (2003), en un primer momento, para cada usuario que llega, se trata de construir un espacio y un tiempo, donde lo central es "búsqueda de la transferencia", a través del cuidado y de un espacio de apaciguamiento posible. Buscar la transferencia ahí donde muchas veces el psicótico aparece no pidiendo ni demandando nada y donde se hace evidente que el diálogo es posible en tanto la disposición queda del lado de quien trata, de quien se dispone a escuchar al sujeto ahí.

En este sentido la institución es pensada y organizada a través del colectivo, en tanto una organización posible de un grupo que posibilita la inscripción en la vida cotidiana, en la posibilidad de responsabilización en la gestión del cotidiano, atendiendo y respetando el surgimiento de la singularidad de cada uno. Esta gestión se orienta a la facilitación de un ambiente lo más rico posible en cuanto a espacios de decir e investir, personas y objetos a encontrar. Espacios susceptibles de transformación y cambios. Podemos plantear entonces que lo terapéutico de la institución es la capacidad de trabajar, de reconocer y articular esta función dispersa y en movimiento, tal como la transferencia que podemos entablar con pacientes en un medio colectivo.

Cómo se traducen estas reflexiones en la práctica:

La cotidianidad en la Comunidad está organizada sobre tres ejes: Ambiente, Lazo e Interno, los cuales están gestionados en las asambleas comunitarias. Gestión en constante diálogo con la institución y el equipo tratante. Diálogo que re-plantea constantemente los objetivos del tratamiento y de los límites de la institución. Lo interno tiene que ver con los talleres grupales (Arte, Mosaico, Literatura, Cine, Voz), los acompañamientos, espacios de escucha individual. Es una franja de tratamiento donde el equipo tiene mayor opinión en tanto muchas de estas acciones son indicadas o propuestas a los usuarios. Este espacio también implica el trabajo de discusión interna y reflexión del equipo tratante, en reuniones de equipo y supervisión. 
La gestión de la asamblea en éste ámbito regula la inscripción y abandono de los cupos en los talleres. Además de llevar el ritmo de los referentes de cada uno de los espacios, es decir lo monitores y tutores que se disponen a trabajar, facilitando la vinculación de los usuarios a los distintos espacios.

Por otro lado, el Lazo implica aquellos grupos y actividades en la Comunidad que ponen en relación a la institución y a los usuarios con el exterior. A través de los Colectivos, se socializa y organiza en grupo la relación a la búsqueda de trabajo, acceso a la vivienda, los intercambios de dinero a través de la venta de objetos producidos en los talleres, los intercambios culturales con otros países, por ejemplo. Además del trabajo del equipo tratante con las reuniones que se realizan al exterior y que tienen por objeto el tratamiento o seguimiento de un usuario en la denominada red asistencial.

Por último, el Ambiente se relaciona a la creación y facilitación de espacios que mantienen el cuerpo institucional; es decir el cuidado del espacio a través del aseo, del jardín, los desayunos y almuerzos; los espacios de intercambio diario que se producen a propósito de los intereses comunes y que se instituyen a través de la asamblea como un asunto colectivo. Como ejemplo, encontramos la gestión de la biblioteca, la función de tesorería (cobranza del dinero de almuerzos, por ejemplo) y la organización del tiempo libre y el ocio.

\section{Discusión}

A lo largo del recorrido realizado en el trabajo, se observa que el avance hacia una política de des-institucionalización implementada por el Plan Nacional de Salud Mental y Psiquiatría, pareciera dar cuenta de una restitución política a través de las nociones de ciudadanía y derechos humanos de los pacientes psiquiátricos.

Si bien es cierto que los efectos de la des-institucionalización restituyen el derecho de ocupar un lugar político dentro de la institucionalidad civil, el foco en los derechos humanos reduce el problema de los efectos de violencia del poder. Los sitúa únicamente en relación a la trasgresión de es- 
tos derechos, a causa de los excesos y vicios propios de una institución que por su misma estructura propicia un trato inhumano. Se configura entonces la idea de que la eliminación de la institución psiquiátrica permitiría la aparición del ejercicio propiamente terapéutico de la psiquiatría.

Los tres ejes analizados, familia, comunidad y red, ponen de manifiesto que en la relación del saber psiquiátrico con su objeto siempre existe una dimensión de poder que la constituye. La constituye tanto en un momento inicial, cuando es necesaria una determinada distribución del poder para la producción de su saber como verdad, pero también la constituye cada vez que esta verdad se pone en juego, ya que supone una relación de objetivación, una disimetría fundamental entre médico y paciente; entre el psiquiatra y su loco. Como muestra Foucault, "la misma operación terapéutica, esa transformación sobre cuya base alguien considerado como enfermo deja de estarlo, sólo puede llevarse a cabo dentro de la distribución reglada del poder" (Foucault, M. 2005, p.17).

La dificultad de la reforma psiquiátrica en Chile pasa por omitir que el ejercicio del poder no sólo se reduce a la violencia como efecto del modelo asilar, sino que se encuentra en el centro mismo del saber médico. En este sentido, toda intervención producida como efecto de este saber-poder, reproduce ciertas prácticas de dominación, estén estas inscritas dentro o fuera del hospital.

La desinstitucionalización implica la creación de una serie de unidades territorializadas que sustituyen la función terapéutica del hospital, situación que le hace suponer a la práctica psiquiátrica que ya no cuenta con ninguna institución. En esta redistribución de las acciones terapéuticas, se producen una serie de pequeñas instancias que, coordinadas por la red de salud, forman una institución de nuevo tipo. El mismo Foucault advertía que los mecanismos disciplinarios tienen cierta tendencia a "desinstitucionalizarse", "a salir de las fronteras cerradas en donde estaban para circular en estado libre, de esta forma, las disciplinas masivas y compactas se descomponen en procedimientos flexibles de control" (Foucault. M. 2002b. p.214).

La red se configura como un lugar sin territorio (sin el territorio físico del hospital), en la que sin duda se cumplen muchas de las funciones que anteriormente se cumplían al interior del hospital psiquiátrico. 
Deleuze (1999) considera que la atención sectorializada propuesta por las reformas psiquiátricas, aquello que el Plan denomina territorialización de la atención, implica una desterritorialización de los aparatos de control social, los que se distribuyen en la extensión de los espacios públicos.

En este sentido, es posible pensar que las reformas psiquiátricas en general, y el Plan Nacional chileno en particular, responden a una necesidad estatal en relación a una redistribución de la economía del poder en las sociedades contemporáneas. Foucault (2000) enmarca este análisis bajo el concepto de biopolitica; una tecnología del poder ya no referida a la disciplina del cuerpo individual, sino a gestión y gobierno de la multiplicidad de los hombres, en la medida en que forman una masa poblacional "afectada por proceso de conjunto que son propios de la vida, como el nacimiento, la muerte, la enfermedad, etcétera" (Foucault, M. 2000, p. 220).

Las políticas de salud mental en los últimos años, más allá de las particularidades terapéuticas, implicarían un problema de la gestión de la salud en la población, a partir de una maximización de recursos políticos y económicos.

En el caso de Chile, la instauración de estas políticas por parte de los gobiernos de la Concertación, enmarcadas en el proceso de retorno a la democracia después de los años de dictadura, se inscriben dentro de la voluntad por implementar un discurso democrático sobre la relación entre la Sociedad Civil y el Estado, donde el fomento de la participación ciudadana y el énfasis en las iniciativas comunitarias se presentan como acciones legitimadoras ante la comunidad internacional de los procesos de modernización y democratización que comienzan a vivirse dentro del país.

Pese a esto, en la práctica, dichos discursos aparecen desarticulados con respecto a sus propios fundamentos. A decir de Sandoval (2002), conceptos como ciudadanía y participación comunitaria se encuentran vaciados de sus contenidos democráticos históricos, apareciendo como meras formas hegemonizadas por un discurso de poder atravesado por las lógicas neoliberales de optimización de la gestión gubernamental.

Lo anterior tiene como efecto que no exista en las prácticas de salud mental algo así como una "racionalidad comunitaria". Lo que hay no es 
más que una serie de prácticas desagregadas con referencia a cierta noción vaga de lo colectivo, donde su relación con algún posible "efecto terapéutico" no es independiente de su vinculación con el discurso médico y la racionalidad de sus prácticas psiquiátricas.

Entonces, aún cuando podemos observar que existiría una discontinuidad en la relación de la psiquiatría con el poder, existe una continuidad epistemológica en la manera que ésta ha tenido de entender la locura. La instalación del llamado modelo biopsicosocial en las políticas públicas se presenta como novedad a la hora de aproximarse a este fenómeno, a través de una serie de "nuevas miradas" que se suman sin poder integrarse y sin poder superar la concepción de enfermedad, entendida como una consecuencia a la presencia de un elemento patógeno al cuerpo y a la subjetividad, y como carencia respecto a un ideal normativo.

Por último, hemos intentado, a la luz de algunos aportes del psicoanálisis y de una experiencia institucional, que hace suyos estos referentes en el trabajo cotidiano con pacientes psicóticos, introducir y discutir el problema de la locura en dos aspectos: por una parte, en términos epistemológicos, es decir, entendida no como enfermedad, sino pensada como una forma de producción subjetiva, susceptible de ser escuchada y tratada; y por otra el reconocimiento de la dimensión del poder constitutivo de las instituciones, introduciéndolo como eje del tratamiento colectivo, tanto al interior de la institución con los pacientes, como en la relación de la institución con el estado.

La experiencia de la Comunidad Terapéutica de Peñalolén descrita en este trabajo, permite evidenciar la manera en que las nociones que el Plan presenta como directrices de acción en el campo de la SM se van traduciendo en los recursos materiales con los que los equipos cuentan para desarrollar sus propuestas. En efecto, las discusión entorno a los derechos humanos invisibiliza la necesidad de discutir el trabajo en términos técnicos, ambigüedad que permite asignar dineros sin tener en cuenta el costo real necesario para concretar un trabajo técnicamente definido como rehabilitación psicosocial, con recursos suficientes para que los equipos (incluidos los psiquiatras en él), puedan sostener un trabajo propiamente comunitario fuera de los márgenes de las instituciones médicas y psiquiátricas. 
Los recursos, al ser una traducción material de ciertos conceptos epistemológicos, siempre son escasos frente a la necesidad de expandir el trabajo a los contextos comunitarios; situación que va moldeando las prácticas posibles de los equipos. Es en este margen incómodo, donde la propuesta de la Comunidad Terapéutica ha buscado sostener un lugar durante todos estos ańos, a través de una apuesta institucional que es fundamentalmente ética y política.

\section{Bibliografía}

Deleuze, G. (1999). Conversaciones. Valencia: Pre-Textos.

Foladori, H. (2005). ¿Existe la Psicología Comunitaria? En Grupalidad. Teoría e Intervención. Santiago:Espiral.

Foucault, M. (2002a). Los Anormales. Curso en el Collège de France (19741975). México D.F: Fondo de Cultura Económica.

Foucault, M. (2000). Defender la Sociedad. Curso en el Collège de France (1975 - 1976). Buenos Aires: Fondo de Cultura Económica.

Foucault, M. (2005). El Poder Psiquiátrico. Curso en el Collège de France (19731974). Buenos Aires: Fondo de Cultura Económica.

Foucault, M. (2002b). Vigilar y Castigar: Nacimiento de la Prisión. Buenos Aires: Siglo Veintiuno.

Montero, M. (2004).Introducción a la Psicología Comunitaria: Desarrollo, Conceptos y Procesos Buenos Aires: Paidos.

Ministerio de Salud (2005), Guia Clínica Primer Episodio de Esquizofrenia. $1^{a}$ Ed. Santiago: Ministerio de Salud.

Ministerio de SAlud (2005), Orientaciones Técnicas para el Tratamiento y Rehabilitación de personas afectadas de Esquizofrenia, en Psiquiatría Comunitaria en Línea. http://www.psiquiatriasur.cl/portal/modules/wfdownloads/singlefile.php?cid $=17 \&$ lid $=242$

Ministerio de Salud (2000), Plan Nacional de Salud Mental y Psiquiatría. En Psiquiatría Comunitaria en Línea. http://www.psiquiatriasur.cl/portal/ modules/weblinks/viewcat.php?cid=3

Minoletti, A. (2005) Cumplir con la Declaración de Caracas...Asegurar la Implementación de los Planes Nacionales de Salud Mental. En Psiquiatría Comunitaria en Línea. http://www.psiquiatriasur.cl/portal/modules/wfdownloads/topten.php?list=hit

Minoletti, A; Zaccaria, A. (2005) Plan Nacional de Salud Mental en Chile: 10 Años de Experiencia. Rev. Panamericana de Salud Pública, Pan. Am. J. Public Health 18(4/5), 2005. En Psiquiatría Comunitaria en Línea. http:// www.psiquiatriasur.cl/portal/modules/wfdownloads/topten.php?list=hit

Pereda, V. (2003) Sobre una experiencia de trabajo con la psicosis. Rev. de Psicoanálisis Objetos Caídos. Año cuarto, No 5, Verano 2003. 
Oury, J. (1998) Libertad de Circulación y espacio del decir. www.cliniquedelaborde.com

Ley 19.966, Del régimen General de Garantias en Salud.

http://www.psiquiatriasur.cl/portal/modules/wfdownloads/topten.php?list=hit

Decreto Supremo No 570. Reglamento para la Internación de las Personas con Enfermedades Mentales y sobre los Establecimientos que las Proporcionan. En Psiquiatría Comunitaria en Línea. http://www.psiquiatriasur.cl/ portal/modules/wfdownloads/topten.php?list=hit

Fontaine, A. (2007) La institución vinculante. Metapsicología de la función hospitalaria. Clínica de la transferencia en institución. Texto inédito presentado en el Coloquio Locura y Lazo Social.

Medina E. (2006) Panorama Histórico de la Salud Mental. En Psiquiatría y Salud Mental para la Atención Primaria (ED) Quijada, M. (Documento de Trabajo, por publicar)

Sandoval, J. (2002). Ciudadanía "Gobierno" de la Subjetividad y Políticas Sociales. Rev. Última Década, No 17 (pp. 161-177). Centro de Investigación y Difusión Poblacional de Achupallas. Viña del Mar, Chile.

Ley No 18.469 "Regula el ejercicio del derecho constitucional a la protección de la salud y crea un régimen de prestaciones de salud”. http://www.supersalud.cl/ normativa/571/fo-article-556.pdf

Saldivia, S; Grandón, P; Sgombich, X; Silva, F (2004) Evaluación de programas de rehabilitación para pacientes con esquizofrenia. En http://www. redsalud.gov.cl/archivos/salud_mental/documentos_relacionados/Rehabilitacion/Estudio_Evaluaci.pdf

Fecha de Recepción de articulo: 01 de Septiembre 2008

Fecha de Aceptación de artículo: 30 de Octubre 2008 
